\title{
INDIRECT CEREBRAL REVASCULARIZATION ON OPTHALMIC ARTERY BY USING A DRUG-ELUTING BALLON FOR SUSPECTED MOYAMOYA DISEASE
}

\author{
Ahmad Sulaiman Alwahdy ${ }^{1}$, Fritz Sumantri Usman ${ }^{2}$ \\ Correspondence: ule842002@yahoo.com \\ ${ }^{1}$ Staff of Neurology, Neurointervention Sub Division, Fatmawati Central General Hospital, Jakarta, Indonesia \\ ${ }^{2}$ Head of Neurointervention Sub Division, Fatmawati Central General Hospital, Jakarta, Indonesia
}

\author{
Article History: \\ Received: July 14, 2021 \\ Accepted: November 3, 2021 \\ Published: January 1, 2022

\section{Cite this as:} \\ Alwahdy AS, Usman FS. Indirect \\ cerebral revascularization on \\ opthalmic artery by using a drug- \\ eluting ballon for suspected \\ moyamoya disease. Malang \\ Neurology Journal; 2022.8:72- \\ 76. DOI: \\ http://dx.doi.org/10.21776/ub.mnj \\ .2022.008.01.15
}

\section{ABSTRACT}

\begin{abstract}
Moyamoya disease (MMD) is a rare idiopathic progressive vaso-occlusive disease causing multiple occlusion of cerebral vessels lead to ischemic stroke. Asian population is the most common race to be affected. We present a male patient 33-years old with suspected MMD with right hemiparesis and neurocognitive changes. On digital substraction angiography (DSA) there was appearance of 'puff of smoke' on his right hemisphere, stenosis middle cerebral arteries M1 bilaterally, stenosis of right opthalmic artery (OA), stenosis of left anterior cerebral artery (ACA) and aplasia of right ACA. Ballon angioplasty was performed on right $\mathrm{OA}$ that supply the contralateral symptomatic stenosis area (left A1) indirectly through anterior ethmoidal artery and anterior falcine artery (OA-ACA collateral). While no guidelines for the management of MMD, cerebral revascularization by using drug-eluting ballon (DEB) in right opthalmica artery is potentially effective treatment that could allow the brain to have good blood supply (gives good collateral to both ACA), reduces burden of the fragile moyamoya vessels to be ruptured followed by improvement of clinical results. Patient's selection by understanding the stage, its progressivity and collateral formation are crucial before decision is made.
\end{abstract}

Keywords: Angioplasty, moyamoya disease, neurocognitive, stroke

\section{Introduction}

Firstly described in 1957 by Takeuchi and Shimizu, moyamoya disease is an occlusive cerebrovascular disease of unknown etiology. Multiple occlusion especially in distal ICA and anterior, middle cerebral arteries lead to development of abnormal collateral vascular network at the base of the brain as compensation for the occlusion. ${ }^{1}$ Prevalency of MMD is more frequent to be found in Asian countries where the onset has a bimodal age distribution. The first peak in early childhood and the second in thirdfourth decade ${ }^{2,3}$ In Washington state and California is reported 0.086 per 100.000 population with female is higher than male. ${ }^{1,4}$

Eventhough MMD etiology is unknown, the progressive stenosis of intimal hyperplasia with intramural thrombus formation thought to be the pathology rather than atherosclerotic changes or inflammation. ${ }^{5}$ The clinical presentations of MMD are various include ischemic stroke, haemorrhagic, seizures, headache, and cognitive impairement. ${ }^{1,6}$ The ischemic are predominantly clinical manifestation although haemorrhagic complications may also occur. Frequently, ishemic symptoms are presents due to stenosis lead to hypoperfusion rather than thromboembolism. In neurocognitive dysfunction of MMD patient, chronic hypoperfusion and haemodynamic compromise are thought to be the underlying mechanism. ${ }^{7,89}$
However, neurocognitive impairement can also occur acutely like in our patient, where his frontal lobe supplied mainly from left ACA, since aplasia of his right ACA were formed due to abnormal formation development during embriologic phase. As a consequence, dominant blood supply of his right frontal side was taken over by right OA and left ACA. Before angioplasty was performed, OA-ACA collaterals were not very prominant. We suggest chronic hypoperfusion in right side was well tolerated and compensated by formation of abnormal collateral vascular network, manifestation of acute neurocognitive changes can be occured when there was no sufficient collateral blood supply formation during newly left ACA stenosis development. There is no known effective medical therapy to prevent progressive stenosis in MMD. Most commonly done were by performing surgical revascularization to reduce and prevent ischemia either directly by artery-tooartery anastomosis such as extracranial-to-intracranial (ECIC) bypass or indirectly by arteriosynangiosis. ${ }^{8,10} \mathrm{~A}$ direct approach to revascularize by dilate the stenotic segments has rarely been reported and also long-term outcomes were not favorable especially when stenting performed that instent restenosis were mainly outcomes. ${ }^{11,12}$ In this case report, we describe the clinical and radiological outcome of patient with MMD who has treated with drug-eluting ballon (DEB) on OA with radiologically and clinically excellent results. 


\section{Case Report}

A 33-year-old Indonesian male was admitted to the hospital for headache, altered mental status and unable to talk or even could not answer simple questions. It was associated with slight weakness on right hand and leg. He has no history of hypertension or other risk factors for vascular diseases to be known. General physical examination in the emergency department showed heart rate $=88 / \mathrm{min}$, blood pressure $130 / 80 \mathrm{~mm} \mathrm{hg}$, temperature- $37.0^{\prime} \mathrm{C}$, respiratory rate $=20 / \mathrm{min}$. Neurological examination showed normal cranial nerve function, motor strength was $4 / 5$ of right arm and leg. He appeared altered, nonverbal, unable to answered questions and looked confused. Initial CT-scan was performed with no signs of bleeding or infarct lessions.

Magnetic resonance imaging (MRI) and DSA was performed and MRI showed remarkable for infarct in left frontal lobe where supplied by ACA (figure 1.a). Brain DSA performed the next day showed multiple stenosis. Moderate stenosis were found in right and left middle cerebral artery (MCA) M1 proximal segment, stenosis on left A1 segment with aplasia on his right A1 with preserved Acom (figure 1.b). After the injection of contrast "puff of smoke" appearance was noticed on right hemisphere (figure 2.A). According to Suzuki and Takaku classification, our patient was on stage three. We also noted that in Suzuki stage III, most cases have already developed OA-ACA collaterals. These collaterals seem to be functional earlier than the duro-pial collaterals developed from the middle meningeal artery. ${ }^{13}$

For basic evaluation of his cognitive symptoms initial minimental state examination (MMSE) and to what extent his cognitive symptoms affect his daily activities, barthel index (BI) activity of daily living (ADL) were performed. ${ }^{14}$ Since there was no guideline for the mangement of MMD, we decided to do revascularization by using DEB on his right OA segment. Under local anesthesia. A 7 Fr guide sheath was placed in the right femoral artery. Using coronary guide wire $180 \mathrm{~cm}$ (Asahi Sion blue,Asahi,Japan), a DEB (Sequent please $2.0 \times 10 \mathrm{~mm}$; B.Braun, Berlin, Germany) was deployed successfully by balloon inflation (figure 2.B). After three months follow up, we did MMSE and BI ADL evaluation with significant progress.

\section{Discussion}

The MMD is often multifocal angiopathy with progression of stenosis/occlusion in intracranial blood vessels. Suspected MMD was diagnosed after excluding of any vascular risk factors for atherosclerotic diseases in our patient. The majority of patients present with ischemic hypoperfusion rather than thromboemboli ischemic. ${ }^{7,8,15}$ Percutaneus balloon angioplasty and/or stenting is less invasive procedure to achieve immediate increase in cerebral blood flow with shorter hospitalization compared to surgical revascularization. ${ }^{12}$ Treatment of MMD should aim not only revascularization but also alleviating symptoms and preventing stroke. There is no known effective medical therapy to prevent progressive stenosis in MMD. Histopathological findings have shown that intimal hyperplasia is the main cause of progressive intracranial artery narrowing. ${ }^{15}$ Therefore, we decided to use DEB that contains paclitaxel that promotes stability of microtubules, and cell replication is inhibited in the G0/G1 and G2/M phases. Inhibition of smooth muscle cell mitosis in arterial wall might reduces intimal hyperplasia. Furthermore, cell motility, shape, and transport between organelles may also be affected. ${ }^{11,17}$

Some case reports that have been published by doing combination angioplasty and stenting without drug eluting effectively restore flow of the cerebral circulation even though they did not mention reoccurrence of stenosis. ${ }^{5}$ Other case study in 2007 was reported by using angioplasty and showed no restenosis after 2 years follow up. ${ }^{2}$ Unfortunately, the balloon that was used either with drug eluting or without drug eluting was not mentioned. The results remain controversial where restenosis and /or occlusion and recurrence of clinical symptoms with an average period of 1.8 months and 3.9 months, respectively, have been reported. ${ }^{12}$

In MMD, compensation by forming a vascular network the so-called "puff of smoke" at the base of the brain representing prominent collateralization. ${ }^{18}$ This formation was the beginning stage and the latest stage will not show any moyamoya vessels or major intracranial vessels are supplied from the extracranial vessels. Suzuki and Takaku have described the six stages of MMD. Stage one is the narrowing of the carotid fork;moyamoya vessels begin to appear in stage two. It would become more prominent in stage three where major vessels in the anterior circulation are stenotic or occluded. Posterior cerebral arteries are the next arteries to be occluded in the stage four. In this stage, collateralization is taking place with extracranial circulation and stage five has changes in between stages four and six. ${ }^{18}$

According to Suzuki and Takaku classification, our patient was on stage three. It showed right side was the first affected blood vessels in progressivity of the disease. Due to right aplasia ACA, cerebral blood flow compensates by forming right moyamoya vessels on same side and OA will be more dominant then OA-ACA collaterals will be formed. We believe this progressivity took years and very well compensated by forming collateralization, therefore, clinical signs was remain asymptomatic. If collaterals do not develop in time, ischemic events would be the result. ${ }^{19,20}$ This theory explaines why in our patient has left anterior circulation ischemic with A1 stenosis but not right circulation where moyamoya vessels were present.

In MRI of our patient, it showed subacute infarct or hypoperfusion of left frontal lobe where frontal regions are the usual causes Among adult patients having MMD with cognitive impairment. ${ }^{21,22}$ As the neurocognitive disturbed it will impact to patient's daily activities and functional outcome. ${ }^{7}$ At the begining of symptoms, MMSE score was $8 / 30$ and BI ADL showed 16/20. After three months of the procedure, showed improvement of his MMSE score 28/30, BI ADL became independent and start doing his daily activities like before.

From angiography many stenosis ocured and according to his clinical symptoms, left side of A1 that supply frontal region was the main problem, since his right A1 was aplasia. Fortunately, Acom in this patients was well preserved and it seems that OA-ACA collaterals from his right side also give supply to anterior circulation. 


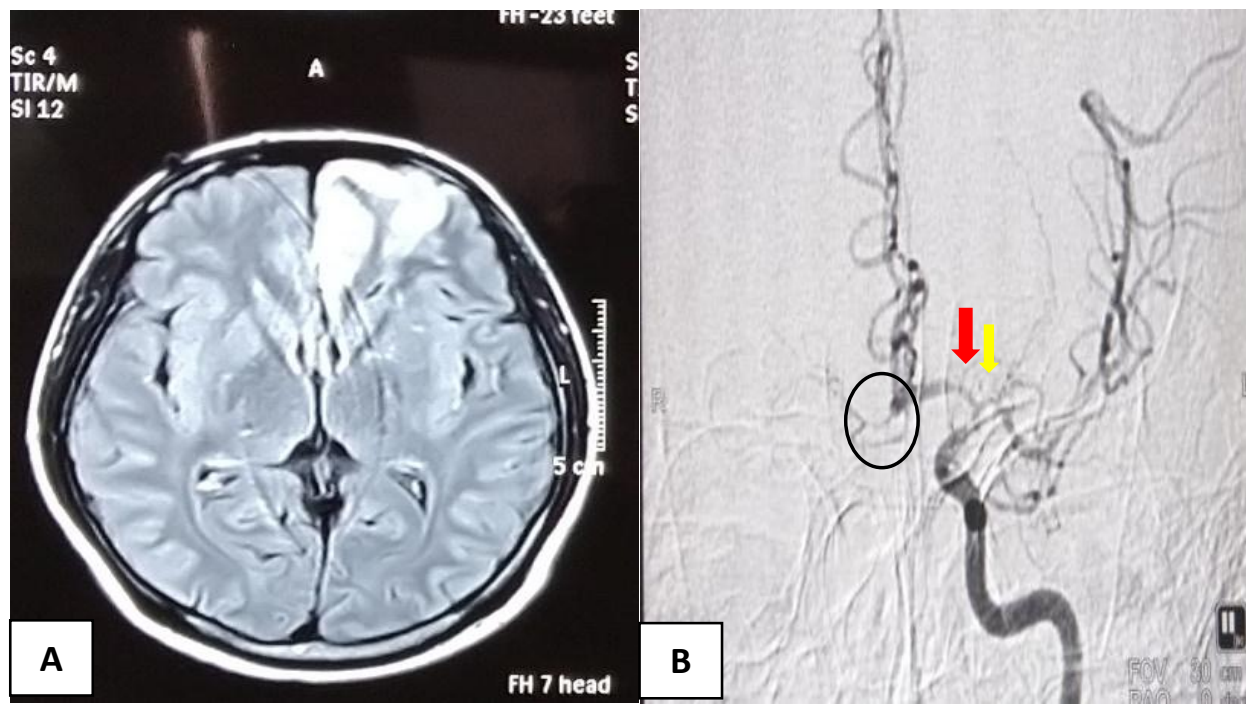

Figure 1. A 33-year old man developed neurocognitive impairement and right hemiparesis. Fluid-attenuated inversion recovery (FLAIR) imaging showed left ACA territory infarction. B.Digital substraction angigraphy (DSA) in anterior-posterior view left side from LICA showed proximal stenosis of A1 (red arrow) and M1 (yellow arrow). Noted OA-ACA collateral from right side through ethmoidal artery can already be seen evnthough not prominent (black circle). ACA: anterior cerebral artery; LICA: Left internal carotid artery; MCA : middle cerebral artery; OA: Opthalmic artery.
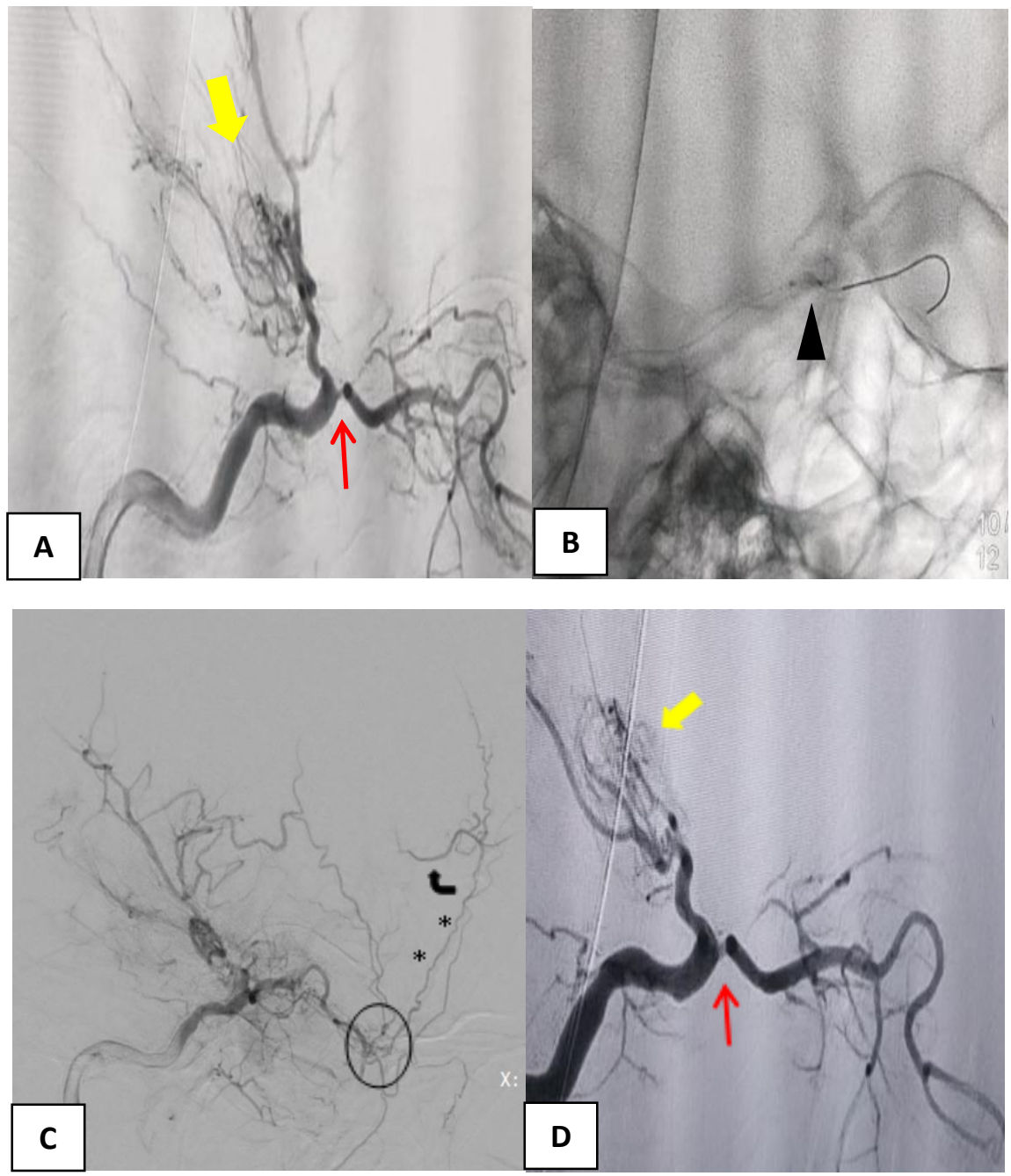

Figure 2. Digital substraction angigraphy (DSA) showed stenosis in the right OA (red arrow) and prominent moyamoya vessels that arrised from MCA (yellow arrow), A. DEB was performed to dilate the stenosis (black triangle, B). After balloon angioplasty procedure, anterior circulation (black curved arrow) were supplied by OA-ACA collateral (black circle) more dominant through anterior ethmoidal artery and anterior falcine artery (black arterisk), C. Furthermore, moyamoya vessels (black arrow) was less prominent and stenosis of right OA were opened (white arrow), D. ACA: anterior cerebral artery; DEB: Drug eluting-ballon; MCA : middle cerebral artery; OA: Opthalmic artery. 
The reasons we choose to dilate the right $\mathrm{OA}$ rather than left A1 and using drug elluting balloon were;(1) It will increase anterior blood circulation from right side to left side (ischemic side) through OA-ACA collaterals through anterior ethmoidalis artery then to anterior falcine artery (figure 2.C);(2) Approximately 30\% of MMD patients present with haemorrhagic stroke secondary to friable collateral vessels and usually occur in the anterior circulation teritory. ${ }^{1,23}$ By dilating the right blood vessels it will reduces the burden of right part of moyamoya vessels (figure 2.D) and decreases the risk to be ruptured in the future;(3) Eventhough the progressivity of the disease could not be stopped, by using DEB will reduces intima hyperplasia for longer time. In our suggestion eventhough stenosis frequently occurs in multiple sites and DEB inhibits progression of stenosis in segment only, increasing the blood supply by dilating the most crucial stenosis would give a good outcome and prevent the burden of moyamoya vessels to be ruptured.

In practice, applying endovascular treatment for MMD has many limitations. First of all, the size of stenosis segment should have enough size for the stent, in this case balloon can be an option. ${ }^{11}$ Another limitations are this is only one single report with succesfull outcomes, larger number of patients and longer term follow-up to evaluate restenosis and clinical manifestations should be evaluated.

\section{Conclusion}

Although this strategy does not treat the underlying cause, it is potentially effective treatment that could allow the brain to have good blood supply, decreases the chance of moyamoya vessels to be ruptured. cerebral revascularization by using drug-eluting ballon (DEB) in right opthalmica artery gives good collateral to both ACA, reduces burden of the fragile moya-moya vessels followed by improvement of clinical results. Patient's selection by understanding the stage, its progressivity and collateral formation are crucial before decision is made. Furthermore, endovascular treatment can also by pass operation time in an urgent setting.

\section{Conflict of Interest}

The authors declare that they have no conflict of interests

\section{Acknowledgement}

It The authors would like to thank to Endovascular Neurointervention team, all staff of neurology department and fellows of neurointervention program of Fatmawati central general hospital.

\section{References}

1. Kim J S. Moyamoya diseases: epidemiology, clinical features, and diagnosis. Journal of Stroke; 2016. 18(1); 2-11.DOI: 10.5853/jos.2015.01627.

2. Rodriguez G J, Kirmani J F, Ezzeddine M A, Qureshi A I. Primary percutaneous transluminal angioplasty for early moyamoya disease. J Neuroimaging; 2007. 17:48-53.DOI: 10.1111/j.1552-6569.2006.00075.x.
3. Suzuki J, Kodama N.Moyamoya disease:A review. Stroke; 1983. 14:104-109. DOI: 10.1161/01. str.14.1.104.

4. Gudepu R K, Qureshi M A, Qureshi I A, Rao L. Case report: A case report of moyamoya disease in a 36 year old African American women. F1000 Research; 2014. 3:297.DOI: 10.12688/f1000research.5859.1.

5. Leonard L L, Michael S, Sandra A, Cornelissen, Pervinder B, Anna S, et al. Angioplasty and stenting of adult onset moya-moya disease. J.inat; 2018. DOI: $10.1016 /$ j.inat.2018.10.013

6. Research Committee on the Pathology and Treatment of Spontaneous Occlusion of the Circle of Willis; Health Labour Sciences Research Grant for Research on Measures for Intrac $\neg$ table Diseases. Guidelines for diagnosis and treatment of moy $\neg$ amoya disease (spontaneous occlusion of the circle of Willis). Neurol Med Chir (Tokyo); 2012. 52:245-266.

DOI: $10.2176 / \mathrm{nmc} .52 .245$

7. Kang C G, Chun M H, Kang J A, Do K H, Choi S J. Neurocognitive dysfunction according to hypoperfusion territory in patients with moyamoya disease. Ann Rehabil Med; 2017. 41(1):1-8.

DOI: $10.5535 / \mathrm{arm} .2017 .41 .1 .1$

8. Penelope D Z, Peter K, Teresa E B, Gary K S, Leslie J D.Neurocognitive performance after cerebral revascularization in adult moyamoya disease. Stroke; 2017. 48:1514-1517. DOI: 10.1161/ STROKEAHA.116.016028

9. Lastri D, Alwahdy A. Clinical and radiologic approach to probable mixed dementia (vascular dementia and progressive supranuclear palsy). Malang Neurology Journal; 2020. 6(1): 46-50. DOI: 10.21776/ub.mnj.2020.006.01.10

10. Baaj AA, Agazzi S, Sayed ZA, Toledo M, Spetzler $R F$, van Loveren $H$. Surgical management of moyamoya disease: A review. Neurosurg Focus; 2009.26:1-7. DOI: 10.3171/2009.01.FOCUS08293

11. Kim T, Kwon O K, Chang W O, Jae S B, Hwang G, Lee $\mathrm{Y}$ J. Intracranial stenting using a drug-eluting stent for moyamoya disease involving suprclinoid internal carotid artery: A case report. Neurol Med Chir; 2014. 54:136-138. DOI: $10.2176 /$ nmc. cr20120272

12. Khan N, Dodd R, Marks MP, Bell-Stephens T, Vavao J, Steinberg GK. Failure of primary percutaneous angioplasty and stenting in the prevention of ischemia in Moyamoya angiopathy. Cerebrovasc Dis; 2011. 31:147-153. DOI: 10.1159/000320253

13. Robert T, CicciÓ G, Sylvestre P, Chiappini A, Weil A G, Smajda S, et al. Anatomic and angiographic analyses of opthalmic artery collaterals in moyamoya disease. AJNR Am J Neuroradiol; 2018. 39:1121-26. DOI: 10.3174/ajnr.A5622.

14. Hariyanti T, Sunarti S, Vistiandini S. Cognitive impairment and depression are the most important risk factors for frailty. Malang Neurology Journal; 2020. 6(1): 20-23. DOI: 10.21776/ub.mnj.2020.006.01.4

15. Kelly ME, Bell-Stephens TE, Marks MP, Do HM, Steinberg GK. Progression of unilateral moyamoya disease: a clinical series. Cerebrovasc Dis; 2006. 22: 109-115. DOI: 10.1159/000093238. 
16. Houkin $\mathrm{K}$, Ito $\mathrm{M}$, Sugiyama $\mathrm{T}$, Shichinohe $\mathrm{H}$, Nakayama N, Kazumata K, et al. Review of past research and current concepts on the etiology of moyamoya disease. Neurol Med Chir (Tokyo); 2012. 52:267-277.DOI:10.2176/nmc.52.267.

17. Gershlick A, Scheerder I D, Chevalier B, Lloyd A S, Camenzind E, Vrints C, et al.Inhibition of restenosis with a paclitaxel-eluting, polymer-free coronary stent. Circulation; 2004. 109:487-493. DOI: 10.1161/01.CIR.0000109694. 8299.A0.

18. Suzuki J, Takaku A. Cerebrovascular 'moyamoya' disease: disease showing abnormal net-like vessels in base of brain. Arch Neurol; 1969. 20:288-299. DOI: 10.1001/archneur.1969.00480090076012

19. Piao R, Oku N, Kitagawa K, Imaizumi M, Matsushita $\mathrm{K}$, Yoshikawa $\mathrm{T}$, et al. Cerebral hemodynamics and metabolism in adult moyamoya disease: Comparison of angiographic collateral circulation. Ann Nucl Med; 2004. 18:115-121. DOI: 10.1007/BF02985101.
20. Taki W, Yonekawa Y, Kobayashi A, Ishikawa M, Kikuchi H, Nishizawa S, et al. Cerebral circulation and metabolism in adults' moyamoya disease: PET study. Acta Neurochir (Wien); 1989. 100:150-154. DOI: 10.1007/BF01403603.

21. Festa JR, Schwarz LR, Pliskin N, Cullum CM, Lacritz L, Charbel FT, et al. Neurocognitive dysfunction in adult moyamoya disease. J Neurol; 2010. 257:806-15. DOI: 10.1007/s00415-009-5424-8.

22. Calviere L, Catalaa I, Marlats F, Januel AC, Lagarrigue J, Larrue V. Improvement in cognitive function and cerebral perfusion after bur hole surgery in an adult with moyamoya disease: Case report. J Neurosurg; 2011. 115:347-9. DOI: $10.3171 / 2011.3 . J N S 101117$

23. Kim SK, Cho BK, Phi JH, Lee JY, Chae JH, Kim KJ, et al. Pediatric moyamoya disease: An analysis of 410 consecutive cases. Ann Neurol; 2010. 68:92-101. DOI: 10.1002/ana.21981. 